\title{
Percutaneous Tibial Nerve Stimulation (PTNS) in Iatrogenic Chronic Vulvar Pain
}

\author{
Pedro Carvalho Moreira, MD, Joana Guimarães, MD, Carina Raposo, BN, Ana Margarida \\ Regalado, MD, and Dalila Veiga, MD
}

Chronic vulvar pain (CVP), although being fairly prevalent, in most cases is not attributable to a specified disorder, i.e., presents as vulvodynia. There are, however, many disorders known to cause CVP, such as radiotherapy and surgery. There have been, so far, no reports in the literature of effective treatment options for latrogenic CVP.

Percutaneous tibial nerve stimulation (PTNS) is a neurostimulation technique used in the treatment of urinary urgency, frequency, urge incontinence, and urinary retention. There has been increasing evidence of its benefits in improving other conditions, such as chronic pelvic pain or sexual dysfunction.

We report a case of a 62 year-old woman with iatrogenic chronic vulvar pain (CVP) submitted to PTNS. The patient reported a consistent and dramatic decrease in both frequency and intensity of pain, assessed by the Brief Pain Inventory
(BPI). A decrease in the pain interference with mood, normal work, and walking/mobility was also noted, as evaluated by BPI and EQ-5D questionnaires. Even though a relapse of symptoms was seen after the end of the treatment protocol, it was possible to achieve more permanent benefits after a maintenance scheme of PTNS, with the patient becoming almost asymptomatic.

Neuromodulation treatments have been reported as effective in cases of vulvodynia, however reports using PTNS are rare. The tibial nerve is easily accessible and provides an optimal site for neurostimulation without the need of an operating room or anesthesia. The overall improvement registered in this case of CVP elicits a new area of research for PTNS. \{Need key words.

Key words: Chronic pain, pelvic pain, vulvar diseases, neuromodulation, percutaneous electric nerve stimulation, tibial nerve
Chronic vulvar pain (CVP), although being fairly prevalent (1), in most cases is not attributable to a specified disorder, i.e., presents as vulvodynia. There is a variety of disorders known to cause CVP, including radiotherapy and surgery (2), yet most of them lack a specific treatment.

Percutaneous tibial nerve stimulation (PTNS) is a neurostimulation technique used in the treatment of urinary urgency, frequency, urge incontinence,

From : Anesthesiology, Intensive Care and Emergency Department,

Centro Hospitalar do, Porto, Porto, Portugal

Author for correspondence: Pedro Carvalho Moreira, MD

Address: Centro Hospitalar do Porto. Anesthesiology, Intensive Care and Emergency Department. Largo Prof. Abel Salazar, 4099-001, Porto, Portugal

E-mail: pedro.aves@gmail.com and urinary retention (3). There has been increasing evidence of its benefits in improving other conditions such as chronic pelvic pain (4) or sexual dysfunction (5).

Sacral neurostimulation is an invasive neuromodulation treatment that has been used for urinary symptoms and fecal incontinence, which has been far more studied than PTNS. However, sacral neurostimulation requires surgical placement of electrodes and thus, less invasive techniques have been developed (6). The tibial nerve is easily accessible and provides an optimal site for neurostimulation without the need of an operating room or anesthesia.

Neuromodulation treatments have been reported as effective in cases of vulvodynia (7-9), however, reports using PTNS are rare. Even more relevant, 
there has been, so far, no reports in the literature of effective treatment options for latrogenic CVP.

\section{CASE REPORT}

A 62 year-old woman, married and working as a geriatric care provider, with Sjogren syndrome and rheumatoid arthritis, was referred to our chronic pain unite (CPU) by her gynecologist with a 3-year history of vulvar pain. Obstetric and gynecological history included 4 pregnancies (2 spontaneous abortions and 2 vaginal deliveries) and a previous history of frequent vaginal infections in adolescence and early adulthood. She had 3 abdominopelvic surgeries in the last 7 years. In 2008 she had a complicated acute diverticulitis, being submitted to a colectomy (Hartmann surgery), cholecystectomy, and right oophorectomy. In 2009, she was diagnosed with a stage II endometrioid adenocarnimoma (10), and treated with total hysterectomy and adjuvant pelvic radiotherapy (total $45 \mathrm{~Gy}$ ) and vaginal brachytherapy ( 3 sessions of $5 \mathrm{~Gy}$ each at a $5 \mathrm{~mm}$ depth). Colorectal anastomosis was performed in the same surgery. As a consequence of radiation-induced enteritis, she had several episodes of bowel obstruction with hospitalization and a surgery for lysis of adhesions in 2010. She has no known drug allergies.

The patient was referred in 2013 to the chronic pain unit (CPU) by her gynecologist with a 3-year history of vulvar pain. Her symptoms developed 3 months after radiotherapy. She had been medicated with paracetamol $1 \mathrm{~g}$, with partial pain relief. No gynecologic disorders were found in the physical examination that could explain her pain. Dermatological, infectious, and neoplastic diseases were excluded.

At the first visit, the symptoms reported by the patient included pain in the vaginal area, the labia majora, and the vestibule. She described a burning sensation, a perception of heaviness, and edema in the vulvar area. Pain was worsened by tight clothing, sitting or orthostatic position, and urination, but not with defecation. Pain was also exacerbated during sexual intercourse, which she avoided completely. Pain relief factors were local cold and supine position. There was no interference with sleep. She also reported a periumbilical pain elicited by efforts with no relation to bowel function and with relief in the supine position. Because of anxiety resulting from this painful condition, she was medicated with estazolam $2 \mathrm{mg}$ and bromazepam $1.5 \mathrm{mg}$. Pain was not interfering with her daily activities. She scored 4 out of 10 at that moment and 8 out of 10 as the worst pain in the previous 7 days in the Numeric Pain Scale (NPS). Labia majora allodynia was identified on physical examination.

Alongside with vulvar pain, the patient complained of recurrent abdominal pain and vomiting crises associated to radiation enteritis relieved with oral butylscopolamine $10 \mathrm{mg}$ and oral tramadol $100 \mathrm{mg}$. She also reported fecal incontinence, with liquid tools during these crises and soiling out of crisis. A stress urinary incontinence was also referred.

The patient was followed during a 6 month period. Tramadol with paracetamol $(37.5 \mathrm{mg} / 325 \mathrm{mg}$ twice a day) was initially tried but was ineffective. Pregabalin $50 \mathrm{mg}$ once a day was prescribed with a good response. NPS scores diminished to 2 out of 10 at the moment and 4 out of 10 as the worst pain in the previous 7 days. The patient was referred back to gynecologist.

Sixteen months later, the patient's gynecologist requested again CPU intervention, reporting failure of conservative medical treatment, as the patient had a progressive worsening of pain. Other causes for vulvar pain were again excluded. Pain was similar in its characteristics, except that it was no longer triggered with urination. She scored 5 out of 10 at that moment and 8 out of 10 as the worst pain in the previous 7 days in the NPS.

Pregabalin was maintained in the same doses and amitriptyline $5 \mathrm{mg}$ daily was initiated, without significant response. The patient was not willing to escalate medical treatment, as she feared its secondary effects.

By May 2015, PTNS became available in our CPU and the patient was enrolled in this treatment. The treatment protocol consisted in 12 sessions of 30 minutes duration, at weekly intervals. The effectiveness of treatment was monitored at the beginning of every session, with a more extended evaluation before the seventh session and at the end of treatment (Table 1).

A 34-gauge needle was placed $1 \mathrm{~cm}$ posterior and $3 \mathrm{~cm}$ proximal to the medial malleolus. Urgent PC( Neuromodulation System (Cogentix Medical, Inc., Minnetonka, MN, USA) was used for stimulation (Fig. 1). Frequency was $20 \mathrm{~Hz}$ and flow wideness was 200 $\mu \mathrm{s}$. The current was gradually increased until a motor 
Table 1. Evaluation of pain characteristics and functional interference evolution throughout treatment.

\begin{tabular}{|c|c|c|c|c|c|c|}
\hline & & & Before PTNS & After 6 sessions & After 12 sessions & \begin{tabular}{|l} 
4th month \\
post-treatment
\end{tabular} \\
\hline \multicolumn{3}{|c|}{ Pain frequency } & Daily & Many times a week & Many times a week & Once a week \\
\hline \multirow{8}{*}{$\mathrm{BPI}^{1}$} & \multirow{4}{*}{$\begin{array}{l}\text { Pain in Last Week } \\
(0-10)\end{array}$} & Worse & 8 & 2 & 4 & 1 \\
\hline & & Least & 0 & 0 & 0 & 0 \\
\hline & & Average & 5 & 1 & 1 & 0 \\
\hline & & Now & 7 & 0 & 1 & 0 \\
\hline & \multicolumn{2}{|l|}{ Treatment Relief } & $40 \%$ & $90 \%$ & $90 \%$ & $100 \%$ \\
\hline & \multirow{3}{*}{$\begin{array}{l}\text { Functional } \\
\text { Interference } \\
(0-10)\end{array}$} & Mood & 8 & 1 & 1 & 0 \\
\hline & & Walking & 4 & 0 & 0 & 0 \\
\hline & & Normal work & 6 & 1 & 1 & 0 \\
\hline \multirow{2}{*}{$\mathrm{HADS}^{2}$} & \multicolumn{2}{|l|}{ Depression } & 5 & 4 & 1 & 7 \\
\hline & \multicolumn{2}{|l|}{ Anxiety } & 10 & 9 & 9 & 9 \\
\hline \multirow{4}{*}{ EQ-5D ${ }^{3}$} & \multirow{3}{*}{$\begin{array}{l}\text { Functional } \\
\text { Interference }^{4}\end{array}$} & Mobility & Some Problems & None & None & None \\
\hline & & Pain/Discomfort & Some Problems & Some Problems & Some Problems & None \\
\hline & & $\begin{array}{l}\text { Anxiety/ } \\
\text { depression }\end{array}$ & Some Problems & Some Problems & Some Problems & Some Problems \\
\hline & Self-rated health & & $60 \%$ & $70 \%$ & $80 \%$ & $60 \%$ \\
\hline \multicolumn{2}{|c|}{ Pain After Session treatment } & & 2 & 0 & 0 & 0 \\
\hline
\end{tabular}

${ }^{1}$ Brief Pain Inventory; ${ }^{2}$ Hospitalar Anxiety-Depression Scale; ${ }^{3}$ EuroQol Group questionnaire. ${ }^{4}$ The patient did not report any interference with other parameters not shown in the table.

response was obtained (the toes flex or the entire foot extends) to determine the appropriate stimulation amplitude and to confirm correct needle placement. The amplitude of current used was between 0.5 and $9 \mathrm{~mA}$. Afterwards, the 30 -minute timed therapy session was started.

The impact of PTNS in the patient's pain and daily activities was evaluated with the Brief Pain Inventory (BPI) (11), the Hospital Anxiety and Depression Scale (HADS) (12), and the EQ-5D questionnaire (13). Results are shown in Table 1 and in Fig. 2.

By the end of the sixth PTNS session, her symptoms were dramatically improved and she had pain-free days. The patient considered a progression from $40 \%$ to $90 \%$ of relief with treatment. Pain intensity was significantly reduced with her worst pain scored as 2 on the NPS. With the continuation of treatments, pain scores consistently decreased (Fig. 1). By the conclusion of the 12 sessions, the patient had a significant decrease in both frequency and intensity of pain. A decrease in the pain interference with mood, normal work, and walking/mobility was also noted, as assessed by the BPI and EQ-5D questionnaires (Table 1). Although not systematically evaluated, the patient reported a benefit in fecal incontinence but not in urinary stress incontinence.

After the 12 sessions, she was evaluated at 2 and 4 weeks post-treatment. A relapse of symptoms was reported during these visits, with an increase in both frequency and intensity of pain. Upon these evaluations, a maintenance scheme of PTNS sessions every 2 weeks was decided for the next 3 months.

Once again, an excellent response to treatment was observed. By the end of the maintenance sessions, the patient was almost asymptomatic. Despite the significant decrease in pain intensity and the clinician's opinion, the patient was unwilling to discontinue her pain medication during this follow-up period, as she thought it could lead to a relapse of symptoms.

The patient experienced a significant reduction in the interference of pain in mood, walking, and normal work throughout the treatment and maintenance period. She also reported a global benefit in her health 


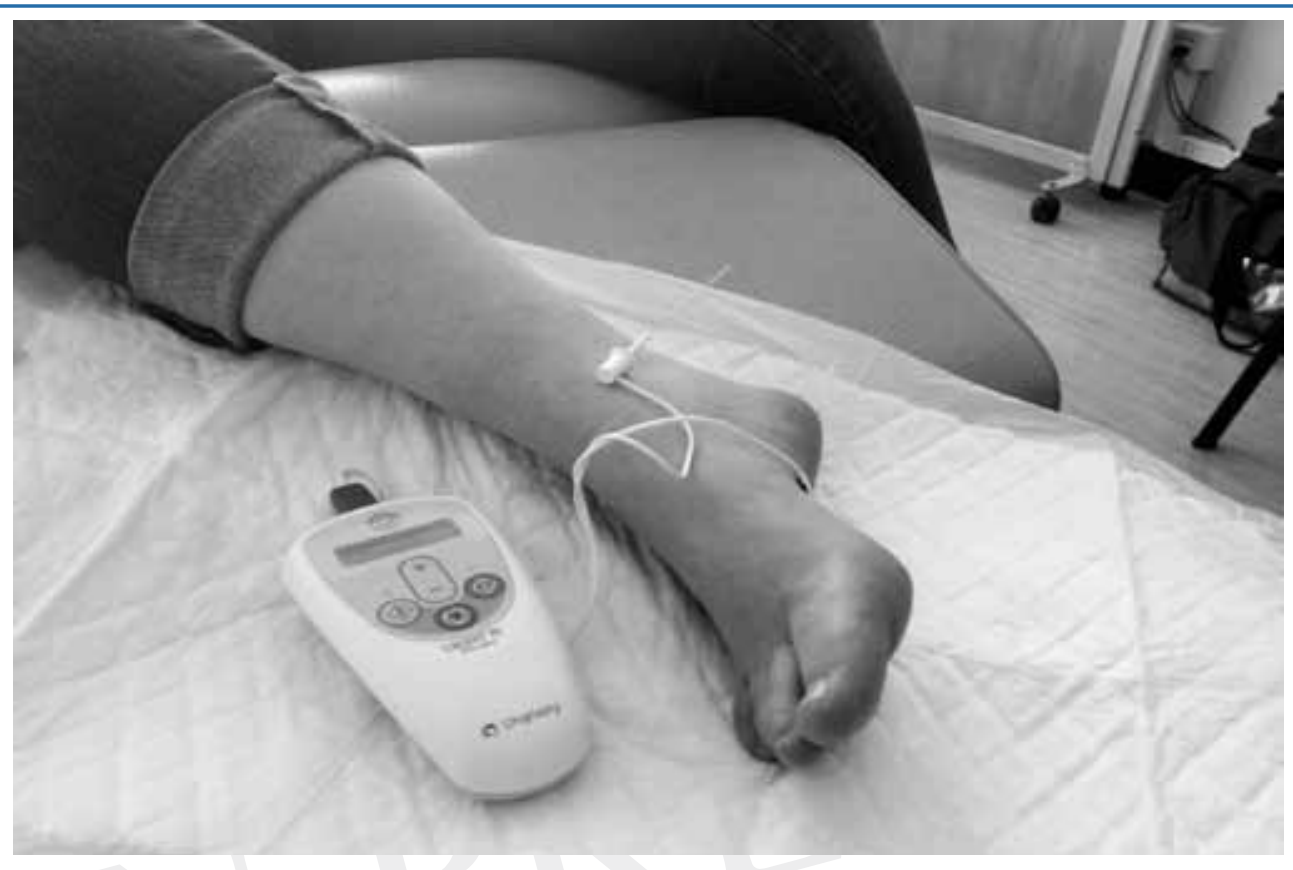

Figure 1. Illustration of the Percutaneous Tibial Nerve Stimulation (PTNS) technique. Patient is in a sitting position with a support for her foot and the foot is positioned in lateral rotation. Needle is placed $1 \mathrm{~cm}$ posterior and $3 \mathrm{~cm}$ proximal to the medial malleolus, attached to the Urgent PC@ Neuromodulation System (Cogentix Medical, Inc. Minnetonka, MN, USA).

and quality of life improvement.

\section{DISCUSSION}

\section{Vulvar Pain - Etiology and Classification}

Persistent vulvar pain terminology has been recently revised, and a consensus classification has been established by the International Society for the Study of Vulvovaginal Disease (ISSVD), the International Society for the Study of Women's Sexual Health (ISSWSH), and the International Pelvic Pain Society (IPPS). Under this consensus, the case here reported is now classified as iatrogenic, as either surgery and/ or radiotherapy were the probable cause of the CVP (2).

Although it differs from vulvodynia, in which there is no identifiable cause for the pain, there are no specific treatments indicated for latrogenic CVP, as literature on this subject is very sparse.

\section{Vulvar Anatomy and Innervation}

The innervation of the external genitalia is mainly by the pudendal nerve, which arises at S2-S4 levels
(Fig. 3). This nerve branches anteriorly to innervate the perineal membrane, external genitalia, and the clitoris, and posteriorly to the inferior rectal nerve, supplying the ischiorectal fossa and the anal area (Fig. 4). Pelvic parasympathetic innervation is also carried through S2 to S4 fibers, passing through the urogenital hiatus and reaching the perineum along the urethra and vagina.

\section{Neuromodulation}

Neuromodulation has been studied for decades. However its mechanism of action is not yet completely understood, many theories exist:

- In lower urinary tract dysfunction, it is thought that neuromodulation causes rebalancing of inhibitory and excitatory impulses in the central nervous system responsible for bladder function (14).

- In regards to the impact of neuromodulation on pain, the gate control theory suggests that stimulation of large somatic fibers modulates the thinner afferent $A C$ or $C$ fibers, thus decreasing pain perception (5).

- Another possible mechanism of action is that 

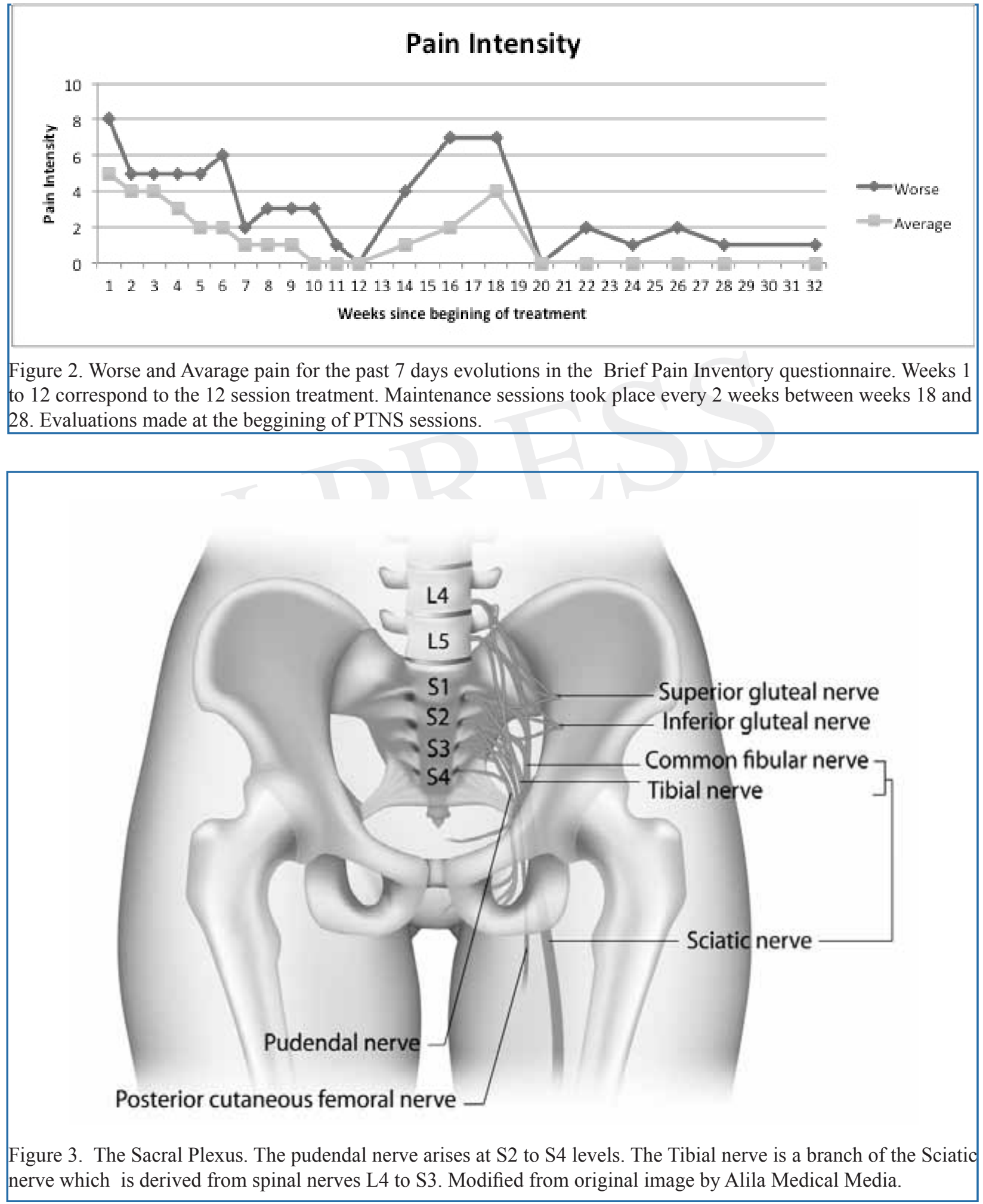


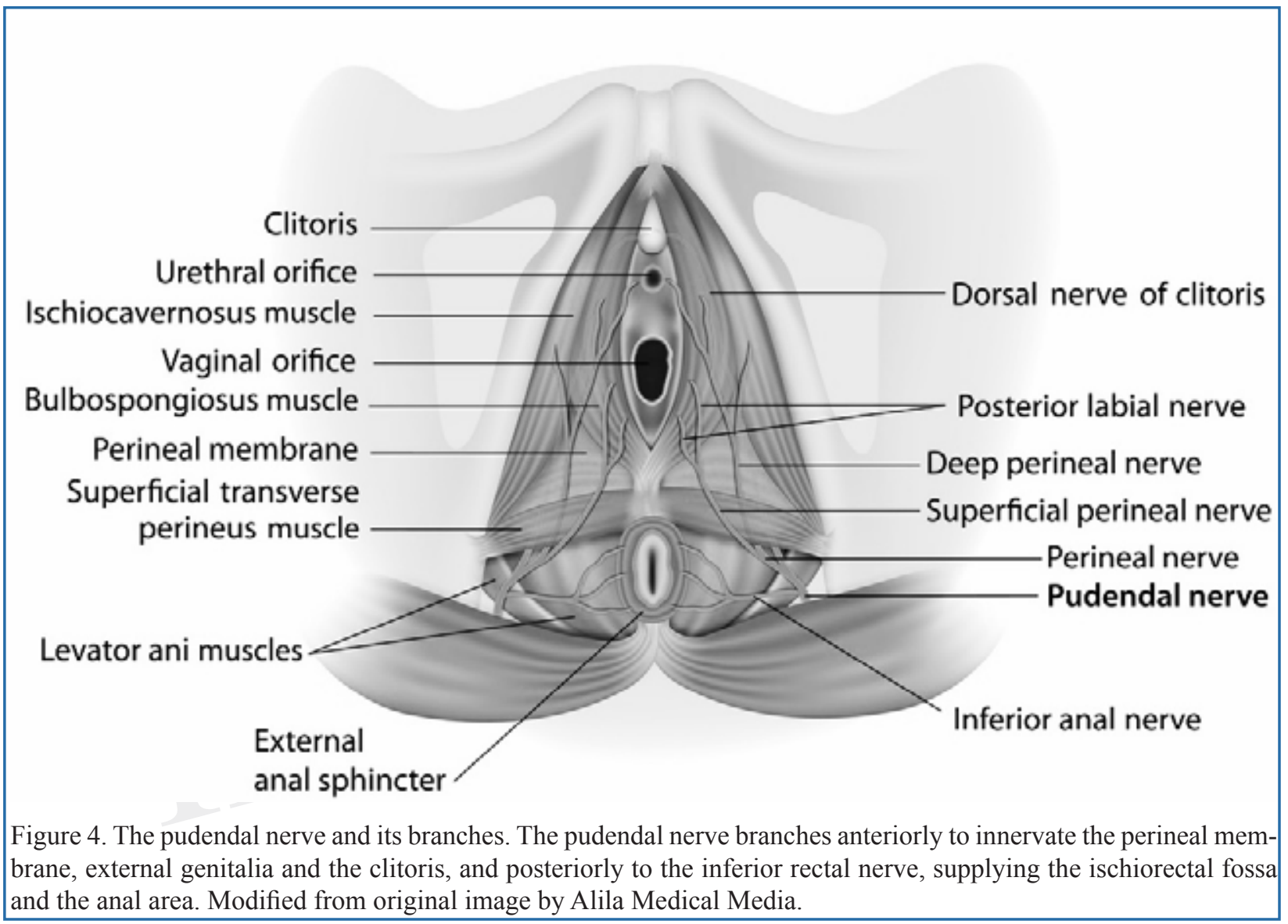

seen in transcutaneous electrical nerve stimulation and acupuncture therapy where nerve stimulation leads to elevation of endorphin levels at the spinal level $(15,16)$.

\section{Percutaneous Tibial Nerve Stimulation}

The tibial nerve is a sensory and motor nerve that originates from spinal roots L4 through S3, which also contribute directly to sensory and motor control of the urinary bladder and pelvic floor. The lure of the tibial nerve is that it is easily accessible with landmarks.

First described in 1983 (17), PTNS is typically performed with patients in the supine position with the knees abducted and the soles of the feet together. A 34-gauge needle is inserted into the skin 3 fingerbreadths cephalad to the medial malleolus. Agrounding pad is placed on the arch of the ipsilateral foot. The amplitude of the stimulation is increased until the first toe curls or the toes fan. This technique has the advantage of being performed without the need of an operating room or anesthesia requirement.

PTNS has been more extensively studied in the treatment of pelvic organ dysfunctions commonly associated with chronic pelvic pain (CPP). Recent reviews show promising results both in lower urinary tract dysfunction $(18,19)$ and fecal incontinence $(20)$, although better quality evidenced is needed.

PTNS impact on CPP has also been addressed in 2 reviews $(4,19)$. With only 2 trials with control groups analyzed $(21,22)$, both reviews concluded for the need of better evidence. Another randomized controlled trial published afterwards (23) has corroborated the promising evidence of the previous studies, although larger studies are needed.

So far, there is no published evidence of PTNS efficacy on vulvar pain conditions, except for a case report of its use in 2 patients with clitoral pain, a sub- 
set of vulvar pain, with resolution of symptoms (24).

There is a lack of consensus on the optimal PTNS regimen, therefore, the investigators opted for the more commonly described regimen, which consists of 30 minute sessions repeated once a week for 12 weeks (20). Even though both motor and sensory responses have been described as good endpoints to confirm correct needle placement, in this case the investigators were able to achieve a motor response in every treatment session.

After initial treatment, PTNS can be repeated as a long-term treatment to maintain achieved benefits (25), but there are currently no recommendations available on subsequent regimens namely their indications (if it should be applied to all patients or only in those in which symptoms relapse) and their fre-

\section{REFERENCES}

1. Reed BD, Harlow SD, Sen A, Legocki LJ, Edwards RM, Arato $\mathrm{N}$, Haefner HK. Prevalence and demographic characteristics of vulvodynia in a population-based sample. Am J Obstet Gynecol 2012; 206:170:e1-e9.

2. Bornstein J, Goldstein A, Coady D. 2015 Consensus terminology and classification of persistent vulvar pain. 2015; http://pelvicpain.org/professional/publications/articles/2015-consensusterminology-and-classification-of-p.aspx

3. Biemans JMAE, van Balken MR. Efficacy and effectiveness of percutaneous tibial nerve stimulation in the treatment of pelvic organ disorders: A systematic review. Neuromodulation 2013; 16:25-33.

4. Tirlapur SA, Vlismas A, Ball E, Khan KS. Nerve stimulation for chronic pelvic pain and bladder pain syndrome: A systematic review. Acta Obstet Gynecol Scand 2013; 92:881-887.

5. Van Balken MR, Vergunst $H$, Bemelmans BLH. Sexual functioning in patients with lower urinary tract dysfunction improves after percutaneous tibial nerve stimulation. Int J Impot Res 2006; 18:470-475.

6. Peters KM. Alternative approaches to sacral nerve stimulation. Int Urogynecol J 2010; 21:1559-1563.

7. De Andres J, Sanchis-Lopez N, Asensio-Samper JM, FabregatCid G, Dolz VM. Peripheral subcutaneous vulvar stimulation in the management of severe and refractory vulvodynia. Obstet Gynecol 2013; 121:495-498.

8. Murina F, Bianco V, Radici G, Felice R, Di Martino M, Nicolin $U$. Transcutaneous electrical nerve stimulation to treat vestibulodynia: A randomised controlled trial. BJOG 2008; 115:11651170.

9. Ramsay LB, Wright JJ, Fischer JR. Sacral neuromodulation in the treatment of vulvar vestibulitis syndrome. Obstet Gynecol 2009; 114:487-489.

10. Pecorelli S. Revised FIGO staging for carcinoma of the vulva, cervix, and endometrium. Int J Gynaecol Obstet 2009; 105:103104. quency. In the case here reported, the investigators decided to administer subsequent monthly treatments in order to sustain the achieved clinical improvement.

\section{CONCLUSIONS}

PTNS is a novel, minimally invasive, neurostimulation modality, which can be easily performed on an outpatient basis. As a recent treatment modality, literature supporting its efficacy in CPP conditions is still sparse, but promising.

The overall improvement registered in this case of CVP, a subset of CPP, elicits a new area of research for PTNS. Further investigation is needed to establish PTNS as a possible treatment modality for CVP.

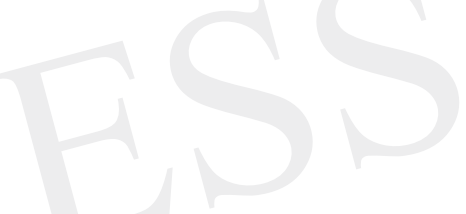

11. Azevedo LF, Pereira AC, Dias C, Agualusa L, Lemos L, Romão J, Patto T, Vaz-Serra S, Abrunhosa R, Carvalho CJ, Cativo MC, Correia D, Correia J, Coucelo G, Lopes BC, Loureiro M do C, Silva B, Castro-Lopes JM. Tradução, adaptação Cultural e estudo Multicêntrico de Validação de instrumentos para rastreio e avaliação do impacto da Dor Crónica. Dor 2007; 15:6-56. [Portuguese]

12. Pais-Ribeiro J, Silva I, Ferreira T, Martins A, Meneses R, Baltar M. Validation study of a Portuguese version of the Hospital Anxiety and Depression Scale. Psychol Health Med 2007; 12:225235.

13. Ferreira LN, Ferreira PL, Pereira LN, Oppe M. The valuation of the EQ-5D in Portugal. Qual Life Res 2014; 23:413-423.

14. Shafik A, Ahmed I, El-Sibai O, Mostafa RM. Percutaneous peripheral neuromodulation in the treatment of fecal incontinence. Eur Surg Res 2003; 35:103-107.

15. Oosterwijk R, Meyler W, Henley E, Scheer S, Tannenbaum J. Pain control with TENS and team nerve stimulators: A review. Crit Rev Phys Rehabil Med 1994; 6:219-258.

16. Chang CJ, Huang S-T, Hsu K, Lin A, Stoller ML, Lue TF. Electroacupuncture decreases $\mathrm{C}$-fos expression in the spinal cord induced by noxious stimulation of the rat bladder. J Urol 1998; 160:2274-2279.

17. McGuire EJ, Zhang SC, Horwinski ER, Lytton B. Treatment of motor and sensory detrusor instability by electrical stimulation. $J$ Urol 1983; 129:78-79.

18. Schneider MP, Gross T, Bachmann LM, Blok BFM, Castro-Diaz D, Del Popolo G, Groen J, Hamid R, Karsenty G, Pannek J, Hoen L 't, Kessler TM. Tibial nerve stimulation for treating neurogenic lower urinary tract dysfunction: A systematic review. Eur Urol 2015; 68:859-867.

19. Gaziev G, Topazio L, lacovelli V, Asimakopoulos A, Di Santo A, De Nunzio C, Finazzi-Agro E. Percutaneous tibial nerve stimulation (PTNS) efficacy in the treatment of lower urinary tract dysfunctions: A systematic review. BMC Urol 2013; 13:61. 
20. George AT, Maitra RK, Maxwell-Armstrong C. Posterior tibial nerve stimulation for fecal incontinence: Where are we? World $J$ Gastroenterol 2013; 19:9139-9145.

21. Gokyildiz S, Kizilkaya Beji N, Yalcin O, Istek A. Effects of percutaneous tibial nerve stimulation therapy on chronic pelvic pain. Gynecol Obstet Invest 2012; 73:99-105.

22. Kabay S, Kabay SC, Yucel M, Ozden H. Efficiency of posterior tibial nerve stimulation in category IIIB chronic prostatitis/ chronic pelvic pain: A sham-controlled comparative study. Urol Int 2009; 83:33-38.

23. Istek A, Gungor Ugurlucan F, Yasa C, Gokyildiz S, Yalcin O.
Randomized trial of long-term effects of percutaneous tibial nerve stimulation on chronic pelvic pain. Arch Gynecol Obstet 2014; 290:291-298.

24. Elkattah R, Trotter-Ross W, Huffaker RK. Percutaneous tibial nerve stimulation as an off-label treatment of clitoral pain. Female Pelvic Med Reconstr Surg 2014; 20:e1-e4.

25. Peters KM, Carrico DJ, Wooldridge LS, Miller CJ, MacDiarmid SA. Percutaneous tibial nerve stimulation for the long-term treatment of overactive bladder: 3-year results of the STEP study. $J$ Urol 2013; 189:2194-2201. 